\title{
BMJ Open Role of systems science in preventing and controlling emerging infectious diseases: protocol for a scoping review
}

Mariam Abdulmonem Mansouri (D) , Frank Kee, Leandro Garcia, Declan T Bradley

To cite: Mansouri MA, Kee F, Garcia L, et al. Role of systems science in preventing and controlling emerging infectious diseases: protocol for a scoping review. BMJ Open 2021;11:e046057. doi:10.1136/ bmjopen-2020-046057

- Prepublication history for this paper is available online. To view these files, please visit the journal online (http://dx.doi. org/10.1136/bmjopen-2020046057).

Received 27 October 2020 Accepted 21 May 2021

Check for updates

(C) Author(s) (or their employer(s)) 2021. Re-use permitted under CC BY-NC. No commercial re-use. See rights and permissions. Published by BMJ.

Centre for Public Health, Queen's University Belfast Belfast, UK

Correspondence to Dr Mariam Abdulmonem

Mansouri;

mmansouri01@qub.ac.uk

\section{ABSTRACT}

Introduction In recent history, many new infectious diseases have affected humans for the first time or have appeared in previously unaffected areas of the world; these diseases are known as emerging infectious diseases (EIDs). Examples of EIDs include COVID-19, Middle East respiratory syndrome and Ebola virus disease. EIDs are known for their complexity. Multiple factors play a role in their spread, including increases in human population, conflicts, urbanisation, air travel, global trade and inequalities in wealth distribution and access to healthcare. In order to gain a better understanding of such complexity, we aim to explore the role of systems science, which allows us to view EIDs in the context of complex adaptive systems rather than simple causes and effects. The objectives of this scoping review are to explore and map the theoretical concepts and key characteristics of studies that use systems methods in controlling EIDs, to identify the gaps in knowledge and disseminate the results. Methods We will follow the Joanna Briggs Institute guidance for this scoping review, comprising the following stages: formulating the research question and subquestions, scanning the literature for available data, selecting relevant publications, charting the data by two independent reviewers, aggregating the findings, reporting, summarising and disseminating the results. We will review peer-reviewed articles, preprints and grey literature available in all languages.

Discussion We intend that this scoping review will contribute to a better understanding of the use of systems methods to inform policymakers about how to prevent and control EIDs

Ethics and dissemination Research ethics approval is not required for a scoping review because it is based on reviewing and collecting data from publicly available sources. To disseminate the findings, results will be shared through academic publications, seminars and conferences.

\section{BACKGROUND}

The WHO defines an emerging infectious disease (EID) as 'one that either has appeared and affected a population for the first time, or has existed previously but is rapidly spreading, either in terms of the number of people getting infected or to new geographical areas'. ${ }^{1}$ Over $60 \%$ of EIDs are zoonotic, having crossed over from animals to humans by a process called spillover. ${ }^{2}$ Recent

\section{Strengths and limitations of this study}

- We explore the use of systems science approaches to the prevention and control of emerging infectious diseases.

- This protocol uses well-recognised methodological frameworks to guide a scoping review.

- The review uses a broad-based search strategy to uncover and map relevant evidence, searching at least six databases and grey literature.

- There will not be a language restriction to data extraction.

- Because of the nature of scoping reviews, no quality assessment of the reviewed data will be performed.

outbreaks due to novel zoonotic infectious diseases include COVID-19, MERS-CoV, Ebola virus disease, Zika virus disease, SARS-CoV and novel influenza viruses. ${ }^{3-5}$ Empirical evidence suggests that many systemic societal and social factors contribute to the appearance of EIDs including urbanisation, climate change, increasing travel and trade, human population growth, human behaviour and the emergence of antimicrobial resistance. ${ }^{46}$

Even though EIDs are known to cause a substantial threat to human life and can impose significant economic burden, Fauci and Morens ${ }^{6}$ argue that the behaviour of such diseases can be predicted and mitigated. ${ }^{6}$ Public health history provides solid evidence that public health measures, including clean water, sanitation, vaccinations and antibiotics, have been effective in decreasing the burden of EIDs. ${ }^{6}$ In more recent history, many countries around the world have invested in increasing national and international communicable diseases control capacities and resources in order to prepare and respond to the next potential pandemic caused by EIDs. ${ }^{7}$ However, due to the novelty of these diseases, there are considerable gaps in our knowledge for the prevention and control of EIDs. ${ }^{45}$

From its early origins in the middle of the last century, systems science has evolved its own 
distinguishable academic identity and a corpus of related scientific literature devoted to the subject. Since then, it has offered academics and practitioners from a variety of fields a number of different approaches and methods for helping better describe and solve 'complex' social, technological, ecological and organisational problems. By 'complex' we mean having features of 'complexity' as found in complex adaptive systems. Those features include multiple interacting components, non-linear relationships and feedback loops between them that might be moderated or synergistic with other system elements. In addition, interactions across multilayered contexts may induce emergent outcomes at various levels that cannot be predicted from relationships observed at any single level. ${ }^{8}$

Those working in this field have appropriated a number of methods and tools ${ }^{9}$ and while many of these have roots in computer science, operational research and simulation, others are alternatively branded as 'soft systems' methods that invoke paradigmatic systems thinking. ${ }^{8}$ This may often take us a long way towards a solution by allowing us to see our problem as a feature of a complex adaptive system rather than focusing on a single cause and effect. ${ }^{8}$ It does so by helping us explore the purpose, behaviour and dynamics of the system's elements and what connects them. ${ }^{10}$ By mapping the patterns of behaviour within a system and what generates them, the use of systems-oriented methods and tools can help us see the 'bigger picture' and better appreciate where the sweet spots for intervention might lie to achieve desirable outcomes. ${ }^{89}$

Systems thinking and the associated tools of systems dynamics have made inroads into healthcare management. ${ }^{9}$ Since many of the big issues affecting public health are easily seen among what are known as 'wicked problems', systems thinking has begun to take root in public health. ${ }^{11}$ For example, Rutter and colleagues ${ }^{12}$ advocated the use of systems science to inform policy debates and to help policymakers find more holistic answers to public health problems. They argued that many public health questions are merely features of complex adaptive systems; therefore, linear or straightforward solutions might not be sustainable,${ }^{12}$ echoing Bircher and Hahn. ${ }^{13-15}$

In 1995, Wilson argued that EIDs represent a complex and dynamic issue, the public health consequences of which are not merely caused by the infectious agent itself. Other key aspects like human behaviour, environmental, geographical and economic factors contribute to the complexity that defies simplistic solutions, ${ }^{16}$ a sentiment which Fauci and Morens echoed in 2012. ${ }^{6}$ Thus, more systemic measures are necessary to address the complexity surrounding EIDs to provide sustainable and adaptive solutions. Thus, such measures might invoke systems-oriented methods, like systems maps, causal loop diagrams and systems dynamics, to demonstrate the links between different elements of a system that might otherwise seem unrelated or be overlooked when studied with conventional methods.
There have been some previous attempts to use systemsoriented approaches in infectious disease research, including, for example, Ebola virus disease, HIV, tuberculosis, influenza and vaccine uptake. ${ }^{17}$ Systems science methods were also recommended by Shi et al, who used examples of disease transmission prediction for influenza A H1N1 in Hong Kong and malaria in Yunnan Province in China, to provide policymakers with a better understanding of how to enhance surveillance and preparedness. ${ }^{18}$ Lee et al provided another example for using systems science, mapping the elements that make up the vaccine manufacturing and distribution system; thereafter, they used multiple systems models to inform policymakers of the best ways to maximise the benefit and reduce wasted resources. ${ }^{19}$ Nevertheless, while interest has been growing, there has been no systematic attempt to scope the charted and uncharted ground over which systems science methods and systems thinking can help societies manage EIDs.

Before writing this protocol, a comprehensive search of databases for scoping or systematic reviews on the topic was conducted. Resources used at this stage included Scopus, the Joanna Briggs Institute (JBI) Database of Systematic Reviews and Implementation Reports, the Cochrane Database of Systematic Reviews, PubMed, Evidence for Policy and Practice Information and Epistemonikos. To the best of our knowledge, no scoping or systematic reviews exploring the role of systems approaches to the control of EIDs have been published (up to the time of writing).

Therefore, this review has the potential to inform researchers and policymakers about innovative ways to prevent and control EIDs at global, national and regional levels. It can provide evidence on how systemsoriented approaches can complement classical public health approaches to the prevention and control of EIDs. Researchers can use this review as a precursor to a more systematic review on the topic.

This review has two objectives. First, to identify the main concepts and characteristics of systems-oriented approaches, as applied to EIDs. Second, to systematically map the evidence for the use of systems-oriented methods in controlling infectious diseases and identify gaps in knowledge.

Following the Population, Concept and Context criteria provided in the JBI framework for clearly delimiting the research question, ${ }^{20}$ this review will focus on human populations; the concept will be systems-oriented methods; while the context is related to EID preparedness and response.

This review will have one main question and multiple subquestions. Those questions might go through further development and change when conducting this study as the reviewers develop a deeper understanding of the topic.

The research question is how can systems-oriented approaches be used for prevention and control of emerging infectious diseases? 
Subquestions include:

- What were the main systems-oriented concepts in these works? Are they defined and operationalised consistently?

- What were the systems-oriented questions?

- What types of elements were considered as part of the system? How were they defined?

- What were the methods used?

- How were stakeholders involved (if any)?

- What are the key insights provoked by the use of the systems-oriented approach?

- What limitations and potential developments for the use of systems methods in controlling EIDs were pointed by other researchers?

\section{METHODS}

After exploring the literature for previous reviews, we had to decide what review methods we should use in order to answer the research question. Scoping reviews are used to map the evidence, including the definition and the main concepts of a topic. ${ }^{20}$ This is in contrast to systematic reviews, which generally answer specific questions or for the assessment of a narrowly defined research question. We decided that scoping review would be the most appropriate method for evidence synthesis in this project, to map the types of available evidence, and identify knowledge gaps, ${ }^{20}$ in line with the objectives of this review.

The JBI framework for scoping reviews was used to write this protocol and will be followed to minimise bias in reporting our review and maintain transparency of the process. JBI is an updated version of the Levac and colleagues' framework developed in 2010, which is based on Arskey and O'Mally's work from 2005. ${ }^{20-22}$ The Preferred Reporting Items for Systematic Reviews and Meta-Analyses extension for Scoping Reviews checklist by Tricco $e t a l^{23}$ will be used to report the review.

\section{Patient and public involvement \\ No patients involved.}

\section{Eligibility criteria}

Since EIDs can appear at a different time in history and spread to new geographical areas, every infectious disease could be considered an EID. ${ }^{1}$ Therefore, to conduct a comprehensive search, we will use the WHO's prioritising diseases for research and development in emergency context list. ${ }^{24}$ Therefore, sources of data considered for this review will not be limited to a specific time frame. This scoping review will include studies conducted to investigate preparedness, prevention and response to EIDs that affect human populations, without limitations to geographical regions or other features such as country income. Also, we will only consider papers that include feedback loops between the factors within the model or network analysis. Moreover, the research team is multinational and fluent in multiple languages, including English, Arabic, Spanish and Portuguese. If we come across data in a language outside our scope, we will refer

\begin{tabular}{|c|c|}
\hline Concept & Search terms \\
\hline Systems approach & $\begin{array}{l}\text { complex }{ }^{*} \text {, systems, systemic, } \\
\text { dynamic }^{*} \text {, agent-based model }{ }^{*}, \\
\text { stochastic simulation, network }^{*} \text {, } \\
\text { compartmental models, multi-agent }\end{array}$ \\
\hline EIDs & $\begin{array}{l}\text { Emerging infectious diseases, } \\
\text { pandemic, coronavirus, Middle East } \\
\text { respiratory syndrome coronavirus }{ }^{*} \text {, } \\
\text { MERS-CoV, COVID-19, severe acute } \\
\text { respiratory syndrome, SARS-CoV-2, } \\
\text { SARS, Ebola, Zika, Nipah, pandemic } \\
\text { influenza*, avian influenza* }\end{array}$ \\
\hline
\end{tabular}

EID, emerging infectious disease.

it to an expert for proper scientific translation. Therefore, there will not be a language limitation to data included in our search. Publications including abstract-only reports and data from commercial websites will be excluded.

\section{Search strategy}

Keeping with JBI recommendations, the search strategy for this review will consist of three steps. ${ }^{20}$ The start date of our scoping review would be on 15 March 2021, we estimate the timeline for presenting our final result to be 12 months which would be by 15 March 2022.

\section{Step 1}

An initial search of published papers using mainly Scopus and Google Scholar will be conducted. The purpose of this limited search is to explore the literature for keywords, Medical Subject Headings keywords and index terms related to the topic. After identifying the terms by the primary reviewer, they will be discussed with the rest of the reviewing team to map the most appropriate terms from the titles, abstracts and main text before moving to the next step in the searching strategy. Examples of keywords to be included in this step are presented in table 1 .

\section{Step 2}

We will the use the keywords agreed by the reviewing team to comprehensively search for eligible peer-reviewed articles and conference papers in Scopus, PubMed and Web of Science. PubMed and Scopus also capture preprints available from arXiv, bioRxiv and medRxiv and those will be included in our search. Grey literature (eg, book chapters, national and international documents) will be identified by snowballing process from the reference list of the selected papers. During this step, we will gain a better understanding of the topic and new keywords might be identified. If this is the case, we will consult the subject librarian about developing the search strategy further.

\section{Step 3}

During this step, we will scan the reference list of the materials included for review. 


\section{Box 1 Draft data extraction fields}

Citation details (author(s), date, title, journal, volume, issue, pages).

- Country.

- Context (EID preparedness and response strategies and/or actions taken).

- Participants/population.

- Details/results extracted from source of evidence.

- Main systems-oriented concepts used and how they were defined and operationalised.

- Systems-oriented questions.

- How the system's boundaries were defined.

- Elements considered as part of the system and their definitions.

Systems-oriented method(s) used.

- Emerging infectious disease(s).

- Key insights and actions provoked using the selected systemsoriented approaches.

- What challenges and potential solutions were raised by authors.

Who are the main stakeholders and how they were involved.

What conclusions were drawn by the researchers.

\section{Screening and selection of sources of evidence}

We will be using Covidence online software for systematic review to organise the screening and data extraction process between the reviewers. The records obtained from all databases will be managed and deduplicated using EndNote reference management software. Titles and abstracts of all papers will be screened by two independent reviewers. Full text of the eligible papers will be screened for inclusion by two independent reviewers. In both stages, disagreements between reviewers about the inclusion of any materials will be resolved by consensus and seeking a third reviewer when needed. This same process will be applied to new sources identified after scanning the reference list of the materials included for review.

\section{Data charting}

The reviewing team will agree on the data extraction framework before the start of the process. Data to be included in the form include, but are not limited to, authors, title, type of material, publication source, systems methods used and challenges raised by the authors (box 1). Thereafter, a pilot testing phase will be conducted by two independent reviewers to maintain consistency with the framework. The same reviewers will execute the data charting independently after the pilot phase. Additionally, we will contact the authors if we need clarification regarding published material.

\section{Analysis of evidence and presentation of the results}

We will qualitatively aggregate and analyse the evidence available in the literature. We will provide a narrative of the use of systems-oriented methods in relation to EIDs and identify gaps in the knowledge where a systematic review or original research is needed. However, this review will not quantify or evaluate the effectiveness of use of those methods in disease control. For example, we will map the types of systems tools, chart the types of systems interventions using the Meadows framework for leverage points ${ }^{25}$ and present the evaluation approaches that have been used in EID modelling, but we will not assess the strengths and weaknesses of those tools or attempt to quantitatively assess the effectiveness of those methods on epidemic prevention and control. The data will be presented using the most appropriate form in tables and charts.

Twitter Mariam Abdulmonem Mansouri @mariammansouri

Contributors MAM wrote the protocol. FK, LG and DTB developed and edited the protocol.

Funding This scoping review is part of a PhD for MAM, funded by Kuwait Civil Commission Service.

Disclaimer The organisation has no role or influence in the design or conduct of the review.

Competing interests None declared.

Patient and public involvement Patients and/or the public were not involved in the design, or conduct, or reporting, or dissemination plans of this research.

Patient consent for publication Not required.

Provenance and peer review Not commissioned; externally peer reviewed.

Open access This is an open access article distributed in accordance with the Creative Commons Attribution Non Commercial (CC BY-NC 4.0) license, which permits others to distribute, remix, adapt, build upon this work non-commercially, and license their derivative works on different terms, provided the original work is properly cited, appropriate credit is given, any changes made indicated, and the use is non-commercial. See: http://creativecommons.org/licenses/by-nc/4.0/.

ORCID iD

Mariam Abdulmonem Mansouri http://orcid.org/0000-0002-7259-2389

\section{REFERENCES}

1 World Health Organization ROfS-EA. A brief guide to emerging infectious diseases and zoonoses 2014 https://apps.who.int/iris/ handle/10665/204722

2 McArthur DB. Emerging infectious diseases. Nurs Clin North Am 2019;54:297-311.

3 Holshue ML, DeBolt C, Lindquist S, et al. First case of 2019 novel coronavirus in the United States. N Engl J Med 2020;382:929-36.

4 Smiley Evans T, Shi Z, Boots M, et al. Synergistic China-US ecological research is essential for global emerging infectious disease preparedness. Ecohealth 2020;17:160-73.

5 Weber DJ, Rutala WA, Fischer WA, et al. Emerging infectious diseases: Focus on infection control issues for novel coronaviruses (Severe Acute Respiratory Syndrome-CoV and Middle East Respiratory Syndrome-CoV), hemorrhagic fever viruses (Lassa and Ebola), and highly pathogenic avian influenza viruses, $\mathrm{A}(\mathrm{H} 5 \mathrm{~N} 1)$ and A(H7N9). Am J Infect Control 2016;44:e91-100.

6 Fauci AS, Morens DM. The perpetual challenge of infectious diseases. N Engl J Med 2012;366:454-61.

$7 \mathrm{Kao} \mathrm{H-Y,} \mathrm{Ko} \mathrm{H-Y,} \mathrm{Guo} \mathrm{P,} \mathrm{et} \mathrm{al.} \mathrm{Taiwan's} \mathrm{experience} \mathrm{in} \mathrm{hospital}$ preparedness and response for emerging infectious diseases. Health Secur 2017;15:175-84.

8 Meadows DH. Thiniking in systems. London, UK: Earthscan, 2009.

$9 \mathrm{NIHR}$, Research SfPH. Developing a systems perspective for the evaluation of local public health interventions United Kingdom: National Institute for health research, 2018. Available: https://sphr. nihr.ac.uk/research/developing-a-systems-perspective-for-theevaluation-of-local-public-health-interventions-theory-methods-andpractice/

10 Ross D, Arnold JPW. A definition of systems thinking: a systems approach. 2015 Conference on Systems Engineering Research: ScienceDirect, Elsevier 2015:669-678.

11 Carey G, Carey N, Joyce A. Systems science and systems thinking for public health: a systematic review of the field. BMJ 2015;5:e009002.

12 Rutter H, Savona N, Glonti K, et al. The need for a complex systems model of evidence for public health. Lancet 2017;390:2602-4. 
13 Bircher J, Hahn EG. Will the Meikirch model, a new framework for health, induce a paradigm shift in healthcare? Cureus 2017;9:e1081.

14 Bircher J, Hahn EG. Applying a complex adaptive system's understanding of health to primary care. F1000Res 2016;5:1672.

15 Bircher J, Hahn EG. Understanding the nature of health: new perspectives for medicine and public health. improved wellbeing at lower costs. F1000Res 2016;5:167.

16 Wilson ME. Travel and the emergence of infectious diseases. Emerg Infect Dis 1995;1:39-46.

17 Chang AY, Ogbuoji O, Atun R, et al. Dynamic modeling approaches to characterize the functioning of health systems: a systematic review of the literature. Soc Sci Med 2017;194:160-7.

18 Liu B, Shi B, Xia S, et al. A complex systems approach to infectious disease surveillance and response. In: Zhong K, ed. International Conference, BHI 2013; Maebashi, Japan, October 29-31, 2013. Proceedings: Springer. Berlin: Springer, 2013.

19 Lee BY, Mueller LE, Tilchin CG. A systems approach to vaccine decision making. Vaccine 2017;35 Suppl 1:A36-42.
20 JB Institute. JBI reviewer's manual, 2019. Available: https://bit.ly/ 3wAivyK

21 Arksey H, O'Malley L. Scoping studies: towards a methodological framework. Int J Soc Res Methodol 2005;8:19-32.

22 Levac D, Colquhoun H, O'Brien KK. Scoping studies: advancing the methodology. Implementation Sci 2010;5:1-9.

23 Tricco AC, Lillie E, Zarin W, et al. PRISMA extension for scoping reviews (PRISMA-ScR): checklist and explanation. Ann Intern Med 2018;169:467-73.

24 (WHO) WHO. Prioritizing diseases for research and development in emergency contexts: WHO, 2021. Available: https://www.who.int/ activities/prioritizing-diseases-for-research-and-development-inemergency-contexts

25 Meadows D. Leverage points: places to intervene in a system, 1999. Available: https://donellameadows.org/archives/leverage-pointsplaces-to-intervene-in-a-system/ 